Article

\title{
Body Mass Index and Risk of Gallbladder Cancer: Systematic Review and Meta-Analysis of Observational Studies
}

\author{
Wenbin Tan ${ }^{1, \dagger}$, Min Gao ${ }^{2, \dagger}, \mathrm{Ning}_{\mathrm{Liu}}{ }^{3}$, Guoan Zhang ${ }^{4}$, Tong $\mathrm{Xu}^{5}$ and Wen Cui ${ }^{4, *}$ \\ Received: 2 August 2015 ; Accepted: 11 September 2015 ; Published: 25 September 2015 \\ 1 Department of Basical Medicine, Jining Medical University, 16 Hehua Road, Jining 272067, China; \\ lzhzsdxsyx@gmail.com \\ 2 Department of Clinical Laboratory, Jining NO.1 People's Hospital, 6 Jiankang Road, Jining 272011, China; \\ medchenjun@126.com \\ 3 Department of Information Technology, Jining Medical University, 16 Hehua Road, Jining 272067, China; \\ liuningsci@126.com \\ 4 Department of Pathology, Jining Medical University, 16 Hehua Road, Jining 272067, China; \\ zga2007@126.com \\ 5 Department of Gastrointestinal Surgery, Affiliated Hospital of Jining Medical University, 79 Guhuai Road, \\ Jining 272029, China; ciwinglee@126.com \\ * Correspondence: cuiwenmd@sina.com; Tel./Fax: +86-0537-3616-216 \\ $\dagger$ These authors contributed equally to this work.
}

\begin{abstract}
OBJECTIVES: To provide a quantitative assessment of the association between excess body weight, interpreted as increased body mass index (BMI), and the risk of gallbladder cancer (GBC). METHODS: We identified eligible studies in Medline and EMBASE up to 1 February 2015 , and reference lists of retrieved articles. Summary relative risks with their $95 \%$ confidence intervals were calculated in a random-effects model. Subgroup analyses were performed according to study design, gender, geographic location, ascertainment of exposure and adjustment for confounders. RESUITS: A total of 12 cohort studies and 8 case-control studies were included in the meta-analysis. Overall, compared with "normal" weight, the summary relative risks of GBC were 1.14 (95\% CI, 1.04-1.25) for overweight individuals (BMI $25-30 \mathrm{~kg} / \mathrm{m}^{2}$ ) and 1.56 (95\% CI, 1.41-1.73) for obese individuals (BMI $>30 \mathrm{~kg} / \mathrm{m}^{2}$ ). Obese women had a higher risk of GBC than men did (women: SRRs 1.67, 95\% CI 1.38-2.02; men: SRRs 1.42, 95\% CI 1.21-1.66), and there was significant association between overweight and GBC risk in women (SRRs 1.26, 95\% CI 1.13-1.40), but not in men (SRRs 1.06, 95\% CI 0.94-1.20). CONCLUSIONS: Findings from this meta-analysis indicate that obesity is associated with an increased risk of GBC, especially in women. Overweight is associated with GBC risk only in women.
\end{abstract}

Keywords: overweight; obesity; body mass index; gallbladder cancer; meta-analysis

\section{Introduction}

Gallbladder cancer (GBC) is a highly fatal malignancy that differs from other cancers of the biliary tract, as being approximately two to five times more common in women than in men [1]. Prognosis of GBC remains poor due to its late clinical presentation, lack of effective non-operative therapy, and rapid turnover [2].

It has been established that history of gallstone is the leading cause of gallbladder cancer worldwide [3]. Additionally, genetic susceptibility, lifestyle factors, smoking, alcohol consumption and diabetes mellitus (DM) also increase the risk of GBC [4-7]. Excess body weight, interpreted as overweight (BMI $25-30 \mathrm{~kg} / \mathrm{m}^{2}$ ) or obesity $\left(B M I>30 \mathrm{~kg} / \mathrm{m}^{2}\right)$, is increasingly recognized as an 
important risk factor for various cancer types. Over the past decades, evidence from clinical studies has addressed the possible link between excess body weight and risk of GBC, but the findings have been somewhat contradictory. Early studies found no statistically significant results [8-10], whereas recent studies did observe a significantly increased risk [11,12].

Our clinical observations indicate a high frequency of obesity among patients with GBC. In the present study, we therefore carried out a systematic review and meta-analysis of all available evidence of observational studies following the meta-analysis of observational studies in epidemiology (MOOSE) guidelines [13] to clarify the association between excess body weight and risk of GBC (Tables S1 and S2).

\section{Materials and Methods}

\subsection{Search Strategies}

Two authors independently performed a literature search using Medline and EMBASE database up to 1 February 2015 with the following text words and/or Medical Subject Heading (MeSH) terms: "body mass index", "BMI", "overweight", "obesity" or "excess body weight", combined with "gallbladder cancer", "gallbladder neoplasm" or "biliary tract cancer". We also reviewed the reference lists of retrieved articles to search for additional studies. No language restrictions were imposed.

\subsection{Study Selection Criteria}

Published articles were included according to the following criteria: (1) the outcome of interest was GBC incidence or mortality; (2) the exposure of interest was overweight or obesity defined by BMI; (3) estimates of odds ratio (OR) or relative risk (RR) with corresponding $95 \%$ confidence intervals (CIs) (or data to calculate them) were reported. Two authors independently evaluated all of the studies retrieved from the databases. Any discrepancies between the two reviewers were solved by joint reevaluation of the manuscript. If there were multiple publications from the same study, the most comprehensive one which could provide detail information for subgroup analysis was selected, using other publications to clarify methodology or characteristics of the population.

\subsection{Data Extraction and Quality Assessment}

Three authors independently evaluated all of the studies retrieved according to the aforementioned inclusion criteria. Discrepancies between the three reviewers were solved by a joint reevaluation of the original article. The following information from each included study was extracted: the first author's last name, geographic location, year of study conducted, sample size, study design, gender and age of participants, duration of follow-up (cohort studies), BMI categories, assessment of BMI (measurement versus self-reported), and the effect estimates with $95 \%$ CIs. When studies provided more than one RR, we extracted all of them and applied the data according to subgroup analysis. The quality of each study was assessed independently by three reviewers using the Newcastle-Ottawa Scale (NOS). The NOS consists of three parameters of quality: selection, comparability, and outcome (cohort studies) or exposure (case-control studies). The NOS assigns a maximum of four points for selection, a maximum of two points for comparability, and a maximum of three points for exposure or outcome [14]. Any discrepancies between reviewers were addressed by a joint reevaluation of the original article. 


\subsection{Statistical Analysis}

To examine associations between overweight/obesity and the risk of GBC, we computed SRRs for two categories of BMI as defined by the World Health Organization (WHO) for adults: overweight (BMI $25-30 \mathrm{~kg} / \mathrm{m}^{2}$ ) and obesity (BMI $>30 \mathrm{~kg} / \mathrm{m}^{2}$ or a discharge diagnosis of obesity) compared with "normal" weight (BMI $18.5-24.9 \mathrm{~kg} / \mathrm{m}^{2}$ ). If studies reported relative risk separately for men and women, we combined the gender-specific estimates to the pooled analysis. When non-standard BMI categories were provided, we selected the category that was most closed to those defined by the WHO. Summary relative risk (SRR) estimates with their corresponding $95 \%$ CIs were combined in a random-effects model. Subgroup analyses were performed according to study design (cohort and case-control studies), gender (men and women), and geographic location (non-Asia and Asia), BMI assessment (measurement and self-reported), Follow-up time ( $>10$ years and $<10$ years), smoking status (smokers and non-smokers), Alcohol abuse (Yes and No). We performed sensitivity analysis to estimate the influence of each individual study on the summary results by repeating the random-effects meta-analysis after omitting one study at a time.

To investigate the sources of heterogeneity across these studies, we performed heterogeneity test, and sensitivity analysis. In heterogeneity test, we used the Cochran $Q$ and $I^{2}$ statistics [15], which were used to test whether the differences found between studies were due to chance. For the $Q$ statistic, a $p$-value of less than 0.10 was considered statistically significant heterogeneity. Publication bias was evaluated using funnel plots and the Egger's test [16]. In the presence of publication bias, we used the "trim and fill" method to correct such bias [17]. Meta-analyses were performed using STATA12.0 (StataCorp., College Station, TX, USA).

\section{Results}

\subsection{Search Results and Study Characteristics}

A total of 883 citations were identified through the literature search. Among the 883 citations, 34 were potentially relevant to the meta-analysis. Among the 34 full text articles, eight studies were not associated with GBC risk, three studies were excluded because gallbladder cancer was not distinguished from extra-hepatic bile duct cancer, and three studies did not provide RR with corresponding CI (or data to calculate them). Finally, a total of 12 cohort studies [8,9,11,18-26] (involving 5101 cases) and 8 case-control studies [10,12,27-32] (involving 1013 cases and 43,591 controls) with data on BMI and/or obesity related to GBC incidence were included in the meta-analysis (Figure 1). The main characteristics of the included studies were summarized in Tables 1 and 2. 15 studies were of high quality (NOS $\geqslant 7$ ). Five studies were of acceptable quality $(\mathrm{NOS}<7)$. 


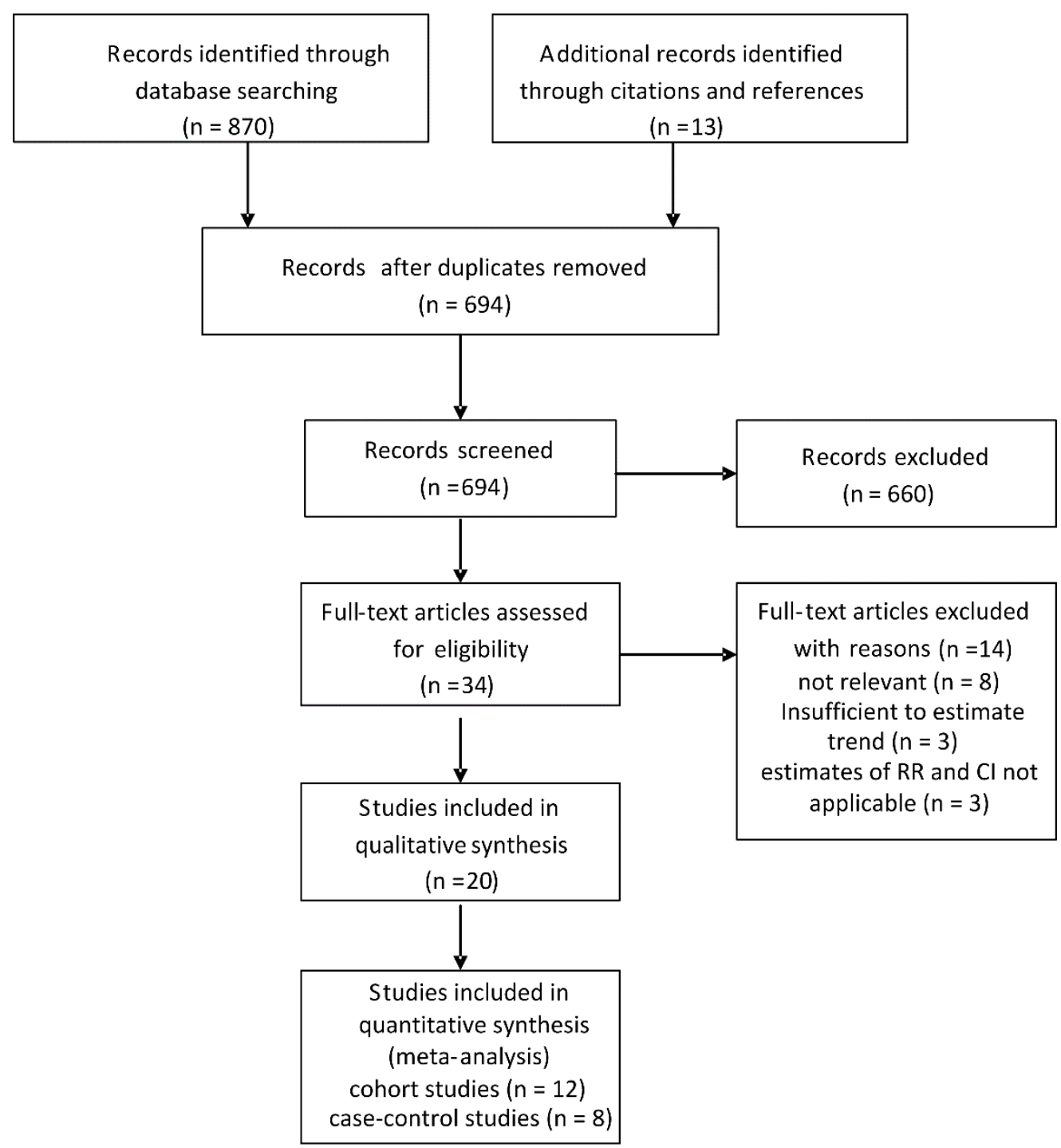

Figure 1. Flow chart of selection of studies included in the meta-analysis.

\subsection{Quantitative Data Synthesis}

As shown in Figure 2A,B, meta-analysis of the 20 studies in a random-effects model found that a statistically significant positive association was observed between BMI and GBC risk (overweight: SRRs $=1.14,95 \% \mathrm{CI}=1.04-1.25, Q=23.85, I^{2}=24.9 \%$; obesity: $\mathrm{SRRs}=1.56,95 \% \mathrm{CI}=1.41-1.73, \mathrm{~m}$, $I^{2}=15.4 \%$ ) compared to "normal weight". We then performed subgroup analyses by study design, gender, geographic location, ascertainment of exposure and adjustment for confounders, as shown in Table 3. 
Table 1. Characteristics of eight case-control studies.

\begin{tabular}{|c|c|c|c|c|c|c|c|c|c|}
\hline \multirow[b]{2}{*}{$\begin{array}{l}\text { Author, Year, Country } \\
\text { (Study Period), Source, } \\
\text { [Reference No.] }\end{array}$} & \multirow[b]{2}{*}{ Case Size } & \multirow[b]{2}{*}{ Controls Size } & \multirow[b]{2}{*}{$\begin{array}{l}\text { Age: Mean } \\
\text { or Range }\end{array}$} & \multirow[b]{2}{*}{$\begin{array}{c}\text { BMI } \\
\text { Ascertainment }\end{array}$} & \multirow[b]{2}{*}{$\begin{array}{c}\text { BMI } \\
\text { Categories }\left(\mathbf{k g} / \mathrm{m}^{2}\right.\end{array}$} & \multicolumn{2}{|c|}{ Effect Estimate $^{a}(95 \%$ CI) } & \multirow[b]{2}{*}{ Adjustments } & \multirow[b]{2}{*}{ NOS } \\
\hline & & & & & & Men & Women & & \\
\hline $\begin{array}{c}\text { Strom, 1995, Mexico and } \\
\text { Bolivia (1984-1988) } \\
\text { Hospital, [10] }\end{array}$ & 65 & 110 & $45-75$ & Self-reported & $\begin{array}{c}<24.0 \\
24.0-25.9 \\
26.0-28.9 \\
>28.0\end{array}$ & $\begin{array}{c}\text { Men and women } \\
1.0 \text { (reference) } \\
1.5(0.5-4.6) \\
2.2(0.7-8.4) \\
1.6(0.4-6.1) \\
\end{array}$ & NA & Age, sex, country & 6 \\
\hline $\begin{array}{c}\text { Zatonski, 1997, Australia, } \\
\text { Canada, The Netherlands } \\
\text { and Poland (1983-1988) } \\
\text { Population, [32] }\end{array}$ & 189 & 1479 & $62.7 \mathrm{~m} 64.2 \mathrm{f}$ & Self-reported & $\begin{array}{l}\text { Quartile 1 } \\
\text { Quartile 2 } \\
\text { Quartile 3 } \\
\text { Quartile 4 }\end{array}$ & $\begin{array}{l}1.0 \text { (reference) } \\
1.0(0.3-3.0) \\
0.7(0.3-2.0) \\
1.0(0.3-2.8) \\
\end{array}$ & $\begin{array}{l}1.00 \text { (reference) } \\
1.70(0.90-3.10) \\
1.50(0.80-3.00) \\
2.10(1.20-3.80) \\
\end{array}$ & $\begin{array}{l}\text { Age, sex, center, } \\
\text { education, alcohol, } \\
\text { smoking, type } \\
\text { of interview }\end{array}$ & 7 \\
\hline $\begin{array}{c}\text { Serra, 2002, Chile } \\
\text { (1992-1995) Hospital, [31] }\end{array}$ & 114 & 114 & $65.8 \mathrm{~m} 70.6 \mathrm{f}$ & Self-reported & $\begin{array}{c}<25.0 \\
25.0-29.9 \\
\geqslant 30.0\end{array}$ & $\begin{array}{c}\text { Men and women } \\
1.0 \text { (reference) } \\
0.8(0.4-1.4) \\
0.9(0.4-1.8) \\
\end{array}$ & NA & Age, sex & 7 \\
\hline $\begin{array}{c}\text { Máchová, 2007, Czech } \\
(1987-2002) \\
\text { Population, [29] }\end{array}$ & 93 & 37772 & $30-64$ & Measured & $\begin{array}{c}18.5-24.9 \\
25-30 \\
\geqslant 30.0\end{array}$ & $\begin{array}{l}1.00 \text { (reference) } \\
1.01(0.24-4.32) \\
0.76(0.08-7.41)\end{array}$ & $\begin{array}{l}1.00 \text { (reference) } \\
1.07(0.58-1.95) \\
0.73(0.36-1.50) \\
\end{array}$ & $\begin{array}{l}\text { Age, smoking, } \\
\text { height, } \\
\text { hypertension }\end{array}$ & 8 \\
\hline $\begin{array}{l}\text { Hsing, 2008, China } \\
\text { (1997-2001) } \\
\text { Population, [28] }\end{array}$ & 365 & 959 & $34-74$ & Self-reported & $\begin{array}{c}<18.5 \\
18.5-22.9 \\
23.0-24.9 \\
\geqslant 25\end{array}$ & $\begin{array}{c}\text { Men and women } \\
0.62 \text { (0.35-1.09) } \\
1.0 \text { (reference) } \\
1.2(0.85-1.68) \\
1.56(1.17-2.10) \\
\end{array}$ & NA & Age, sex, education & 6 \\
\hline $\begin{array}{l}\text { Grainge, 2009, United } \\
\text { Kingdom (1987-2002) } \\
\text { Population, [27] }\end{array}$ & 86 & 3007 & 72 & Measured & $\begin{array}{c}<25 \\
25-29.9 \\
\geqslant 30.0\end{array}$ & $\begin{array}{c}\text { Men and women } \\
1.00 \text { (reference) } \\
1.03(0.62-1.72) \\
1.51(0.83-2.75) \\
\end{array}$ & NA & $\begin{array}{l}\text { Smoking, alcohol, } \\
\text { NSAID use }\end{array}$ & 8 \\
\hline $\begin{array}{c}\text { Nakadaira, 2009, } \\
\text { Hungary (2003-2006) } \\
\text { hospital, [30] }\end{array}$ & 41 & 30 & $40-69$ & NA & $\begin{array}{c}\leqslant 24.9 \\
25.0-29.9 \\
\geqslant 30.0\end{array}$ & $\begin{array}{c}\text { Men and women } \\
1.00 \text { (reference) } \\
1.5(0.4-5.0) \\
0.8(0.3-1.8) \\
\end{array}$ & NA & Age & 7 \\
\hline $\begin{array}{l}\text { Alvi, 2011, Pakistan } \\
\text { (1988-2007) hospital, [12] }\end{array}$ & 60 & 120 & $18-75$ & Measured & $\begin{array}{l}<23 \\
>23\end{array}$ & $\begin{array}{c}\text { Men and women } \\
1.00 \text { (reference) } \\
1.98(0.62-6.28) \\
\end{array}$ & NA & $\begin{array}{l}\text { Sex, hypertension, } \\
\text { diabetes, smoking }\end{array}$ & 7 \\
\hline
\end{tabular}

NA data not applicable; $\mathrm{m}$, male; $\mathrm{f}$, female; ${ }^{\mathrm{a}}$ relative risks are rate ratios, odds ratios, or standardized incidence ratios. 
Table 2. Characteristics of 12 cohort studies.

\begin{tabular}{|c|c|c|c|c|c|c|c|c|c|c|}
\hline \multirow{2}{*}{$\begin{array}{l}\text { Author, Year, Country, } \\
\text { (Study Period) [Ref. No.] }\end{array}$} & \multirow{2}{*}{$\begin{array}{l}\text { Total } \\
\text { Cohort }\end{array}$} & \multirow{2}{*}{$\begin{array}{l}\text { Age: Mean } \\
\text { or Range }\end{array}$} & \multirow{2}{*}{ Cases } & \multirow{2}{*}{$\begin{array}{l}\text { Follow-up, } \\
\text { Years }\end{array}$} & \multirow{2}{*}{$\begin{array}{c}\text { BMI } \\
\text { Ascertainment }\end{array}$} & \multirow{2}{*}{$\begin{array}{c}\text { BMI } \\
\text { Categories }\left(\mathrm{kg} / \mathrm{m}^{2}\right)\end{array}$} & \multicolumn{2}{|c|}{ Effect Estimate $^{a}(95 \%$ CI $)$} & \multirow{2}{*}{ Adjustments } & \multirow{2}{*}{ NOs } \\
\hline & & & & & & & Men & Women & & \\
\hline $\begin{array}{c}\text { Moller, 1994, Denmark } \\
(1977-1987),[9]\end{array}$ & 43965 & $50 \mathrm{~m} 60 \mathrm{f}$ & 28 & 5 & $\begin{array}{l}\text { Discharge } \\
\text { diagnosis }\end{array}$ & $\begin{array}{c}\text { Non-obese } \\
\text { Obese }\end{array}$ & $\begin{array}{c}1.00 \text { (reference) } \\
0.50(0.1-1.8) \\
\end{array}$ & $\begin{array}{c}1.00 \text { (reference) } \\
1.40(0.9-2.1) \\
\end{array}$ & Age & 6 \\
\hline $\begin{array}{c}\text { Wolk, 2001, Sweden } \\
(1965-1993),[8]\end{array}$ & 28129 & 46.1 & 31 & 10.3 & $\begin{array}{l}\text { Discharge } \\
\text { diagnosis }\end{array}$ & $\begin{array}{l}\text { Non-obese } \\
\text { Obese }\end{array}$ & $\begin{array}{c}1.00 \text { (reference) } \\
0.90(0.1-3.4)\end{array}$ & $\begin{array}{c}1.00 \text { (reference) } \\
1.70(1.1-2.5)\end{array}$ & Age, calendar year & 7 \\
\hline $\begin{array}{l}\text { Calle, 2003, United States } \\
\quad(1982-1998),[18]\end{array}$ & 900053 & 57 & 484 & 16 & Self-reported & $\begin{array}{l}18.5-24.9 \\
25.0-29.9 \\
30.0-34.9\end{array}$ & $\begin{array}{l}1.00 \text { (reference) } \\
1.34(0.97-1.84) \\
1.76(1.06-2.94)\end{array}$ & $\begin{array}{l}1.00 \text { (reference) } \\
1.12(0.86-1.47) \\
2.13(1.56-2.90)\end{array}$ & $\begin{array}{l}\text { Age, race, marital } \\
\text { status, smoking, } \\
\text { aspirin, alcohol, } \\
\text { estrogen therapy }(\mathrm{w})\end{array}$ & 8 \\
\hline $\begin{array}{l}\text { Samanic, 2004, United } \\
\text { States (1969-1996), [25] }\end{array}$ & $\begin{array}{c}4500700 \\
\mathrm{~m}\end{array}$ & $\begin{array}{l}52.18 \text { whites } \\
47.63 \text { blacks }\end{array}$ & $338 \mathrm{~m}$ & 12 & $\begin{array}{l}\text { Discharge } \\
\text { diagnosis }\end{array}$ & $\begin{array}{c}\text { Non-obese } \\
\text { Obese }\end{array}$ & $\begin{array}{c}1.00 \text { (reference) } \\
1.63(1.10-2.41)^{\mathrm{b}}\end{array}$ & NA & Age, calendar year & 6 \\
\hline $\begin{array}{c}\text { Engeland, 2005, Norway } \\
\text { (1963-2001), [19] }\end{array}$ & 2001719 & $20-74$ & 1,715 & 13 & Measured & $\begin{array}{c}18.5-24.9 \\
25.0-29.9 \\
\geqslant 30.0\end{array}$ & $\begin{array}{l}1.00 \text { (reference) } \\
1.00(0.84-1.17) \\
1.38(1.01-1.89)\end{array}$ & $\begin{array}{l}1.00 \text { (reference) } \\
1.27(1.10-1.47) \\
1.88(1.60-2.21)\end{array}$ & Age, birth cohort & 7 \\
\hline $\begin{array}{l}\text { Kuriyama, 2005, Japan } \\
\quad(1984-1992), \text { [22] }\end{array}$ & 27539 & $\geqslant 40$ & 33 & 9 & Self-reported & $\begin{array}{c}18.5-24.9 \\
25.0-27.4 \\
27.5-29.9 \\
\geqslant 30.0\end{array}$ & $\begin{array}{l}1.00 \text { (reference) } \\
0.46(0.05-3.93)\end{array}$ & $\begin{array}{l}1.00 \text { (reference) } \\
0.83(0.23-2.98) \\
3.43(1.19-9.94) \\
4.45(1.39-14.23)\end{array}$ & $\begin{array}{l}\text { Age, smoking, health } \\
\text { insurance, alcohol }\end{array}$ & 7 \\
\hline $\begin{array}{l}\text { Oh, 2005, Korea } \\
\text { (1992-2001), [23] }\end{array}$ & $\begin{array}{c}781283 \\
\mathrm{~m}\end{array}$ & $\geqslant 20$ & 182 & 10 & Measured & $\begin{array}{l}21.0-22.9 \\
23.0-24.9 \\
25.0-26.9 \\
27.0-29.9\end{array}$ & $\begin{array}{l}1.00 \text { (reference) } \\
1.55(1.10-2.20) \\
1.15(0.74-1.80) \\
1.25(0.70-2.24)\end{array}$ & NA & $\begin{array}{l}\text { Age, smoking, } \\
\text { alcohol, } \\
\text { exercise, region }\end{array}$ & 7 \\
\hline $\begin{array}{l}\text { Samanic, 2006, Sweden } \\
\text { (1971-1999), [24] }\end{array}$ & $\begin{array}{c}362552 \\
\mathrm{~m}\end{array}$ & 34.3 & 109 & 19 & Measured & $\begin{array}{c}18.5-24.9 \\
25.0-29.9 \\
\geqslant 30.0\end{array}$ & $\begin{array}{c}1.0 \text { (reference) } \\
0.93(0.62-1.39) \\
1.40(0.73-2.70)\end{array}$ & NA & Age, smoking & 8 \\
\hline $\begin{array}{l}\text { Ishiguro, 2008, Japan } \\
(1994-2004),[20]\end{array}$ & 101868 & $40-69$ & 90 & 10.9 & Self-reported & $\begin{array}{c}\leqslant 22.9 \\
23.0-24.9 \\
25.0-26.9 \\
\geqslant 27.0\end{array}$ & $\begin{array}{l}1.00 \text { (reference) } \\
0.74(0.28-1.92) \\
1.26(0.48-3.33) \\
1.39(0.45-4.34)\end{array}$ & $\begin{array}{l}1.00 \text { (reference) } \\
0.47(0.22-0.98) \\
0.62(0.29-1.34) \\
0.94(0.48-1.88)\end{array}$ & $\begin{array}{l}\text { Age, gender, study } \\
\text { area, diabetes, } \\
\text { smoking, alcohol }\end{array}$ & 6 \\
\hline $\begin{array}{r}\text { Jee, 2008, Korean } \\
\text { (1992-2006), [21] }\end{array}$ & 1213829 & $45.0 \mathrm{~m} 49.4 \mathrm{f}$ & 1882 & 10.8 & Measured & $\begin{array}{c}23.0-24.9 \\
25.0-29.9 \\
\quad \geqslant 30\end{array}$ & $\begin{array}{l}1.00 \text { (reference) } \\
0.97(0.86-1.10) \\
1.65(1.11-2.44)\end{array}$ & $\begin{array}{l}1.00 \text { (reference) } \\
1.27(1.02-2.12) \\
1.44(0.98-2.12)\end{array}$ & Age, smoking & 8 \\
\hline $\begin{array}{l}\text { Song, 2008, Korean } \\
\text { (1994-2003), [26] }\end{array}$ & $170481 \mathrm{f}$ & 55.9 & 181 & 8.75 & Measured & $\begin{array}{c}21.0-22.9 \\
23.0-24.9 \\
25.0-26.9 \\
27.0-29.9 \\
\quad \geqslant 30\end{array}$ & NA & $\begin{array}{l}1.00 \text { (reference) } \\
1.06(0.62-1.80) \\
1.30(0.76-2.22) \\
1.86(1.09-3.18) \\
2.10(0.97-4.51)\end{array}$ & $\begin{array}{c}\text { Age, height, } \\
\text { smoking, alcohol, } \\
\text { exercise, pay level }\end{array}$ & 7 \\
\hline $\begin{array}{l}\text { Hemminki, 2011, Sweden } \\
\text { (1964-2006), (11) }\end{array}$ & 30020 & NA & 28 & 11.2 & $\begin{array}{l}\text { Discharge } \\
\text { diagnosis }\end{array}$ & $\begin{array}{l}\text { Non-obese } \\
\text { obese }\end{array}$ & $\begin{array}{c}\text { Men and women } \\
1.00 \text { (reference) }^{-} \\
1.73(1.16-2.57)^{c}\end{array}$ & NA & $\begin{array}{l}\text { Age, sex, region, } \\
\text { economic status }\end{array}$ & 7 \\
\hline
\end{tabular}

NA data not applicable; $\mathrm{m}$, male; f, female; ${ }^{\text {a }}$ relative risks are rate ratios, odds ratios, or standardized incidence ratios; ${ }^{\mathrm{b}}$ combined whites and blacks; ${ }^{\mathrm{c}}$ combined obesity and family obesity. 


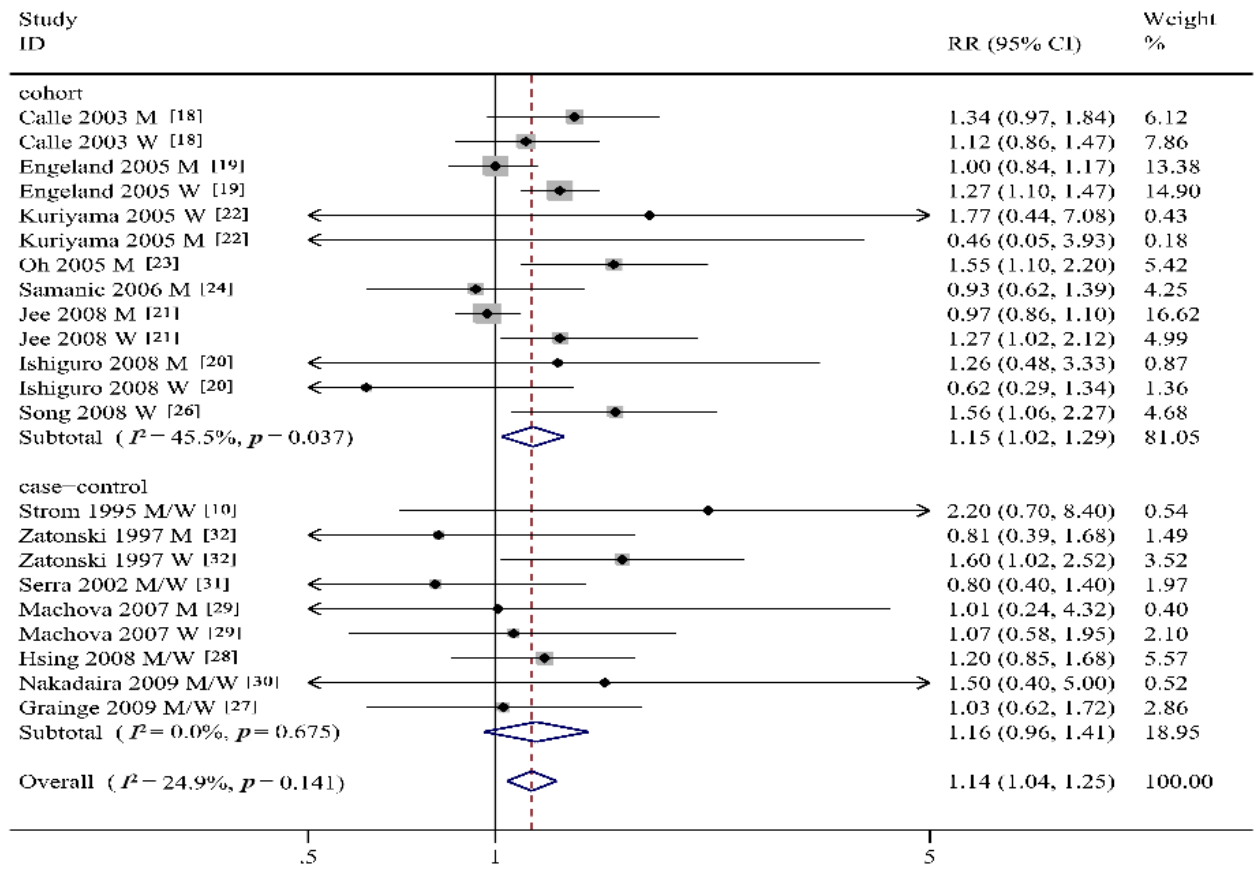

(A)

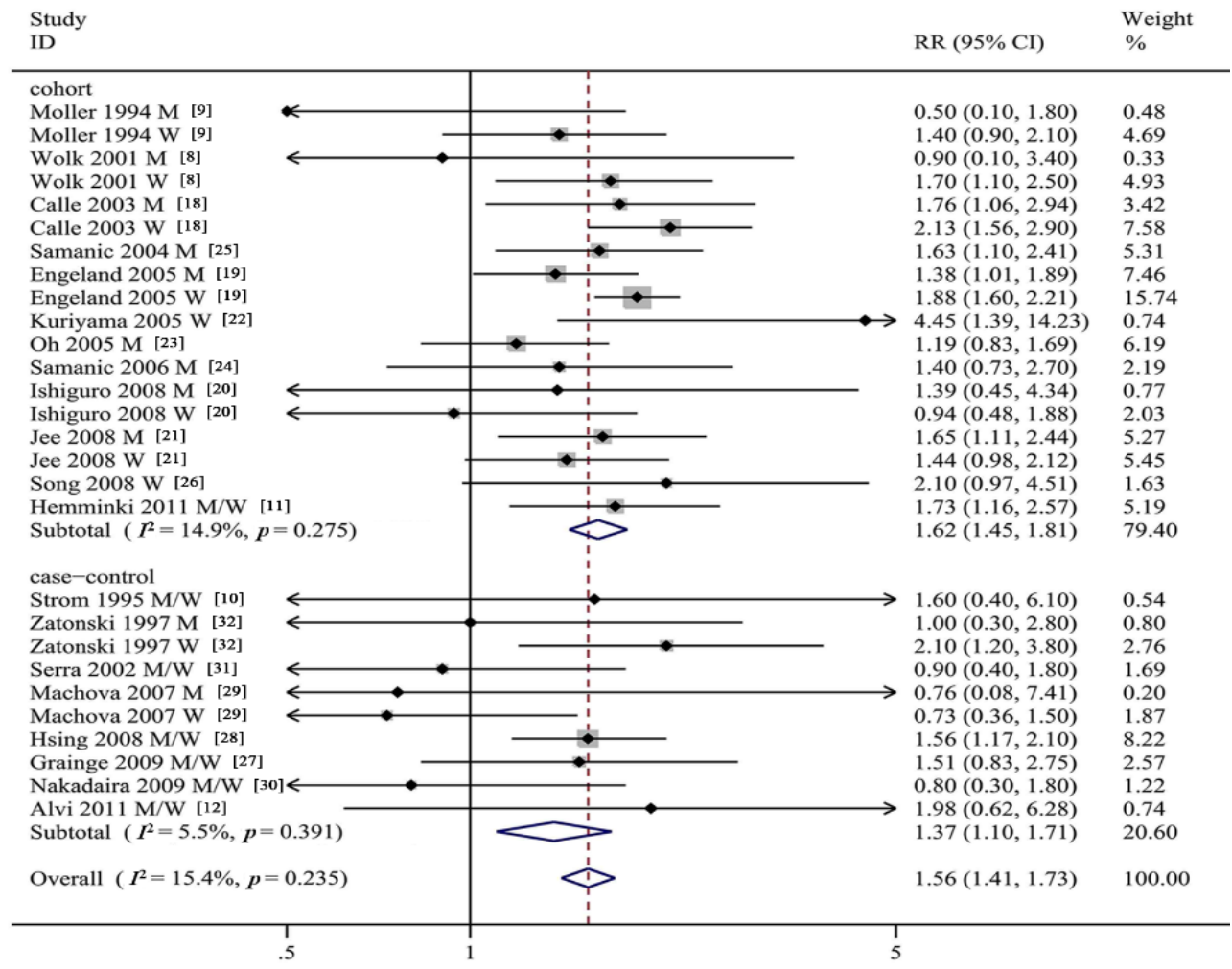

(B)

Figure 2. (A) Forest plot of risk of gallbladder cancer associated with overweight in general population; (B) Forest plot of risk of gallbladder cancer associated with obesity in general population. $\mathrm{M}$, men; $\mathrm{W}$, women; RR, relative risk. 
Table 3. Subgroup analysis of relative risks for association between body mass index (BMI) and gallbladder cancer risk.

\begin{tabular}{|c|c|c|c|c|c|c|c|c|c|c|}
\hline & \multicolumn{5}{|c|}{ Overweight } & \multicolumn{5}{|c|}{ Obesity } \\
\hline & Studies, $n$ & RR (95\% CI) & $p_{\mathrm{h}}$ & $Q$ & $I^{2}, \%$ & Studies, $n$ & RR (95\% CI) & $p_{\mathrm{h}}$ & $Q$ & $I^{2}, \%$ \\
\hline \multicolumn{11}{|l|}{ Study design } \\
\hline Cohort studies & 12 & $1.15(1.02-1.29)$ & 0.04 & 22.03 & 45.5 & 12 & $1.62(1.45-1.81)$ & 0.28 & 19.98 & 14.9 \\
\hline Case-control studies & 8 & $1.16(0.96-1.41)$ & 0.68 & 5.75 & 0 & 8 & $1.37(1.10-1.71)$ & 0.39 & 9.52 & 5.5 \\
\hline \multicolumn{11}{|l|}{ Follow-up time } \\
\hline$>10$ years & 6 & $1.12(1.00-1.27)$ & 0.04 & 17.78 & 49.4 & 9 & $1.65(1.49-1.83)$ & 0.40 & 13.58 & 4.3 \\
\hline$<10$ years & 2 & $1.52(1.06-2.19)$ & 0.54 & 1.22 & 0 & 3 & $1.69(0.91-3.17)$ & 0.10 & 6.32 & 52.5 \\
\hline \multicolumn{11}{|l|}{ Control source } \\
\hline Population & 4 & $1.18(0.96-1.46)$ & 0.67 & 3.19 & 0 & 4 & $1.43(1.09-1.89)$ & 0.30 & 6.12 & 18.3 \\
\hline \multicolumn{11}{|l|}{ Sex } \\
\hline Men & 9 & $1.06(0.94-1.20)$ & 0.24 & 10.33 & 22.5 & 11 & $1.42(1.21-1.66)$ & 0.85 & 5.63 & 0 \\
\hline Women & 8 & $1.26(1.13-1.40)$ & 0.45 & 6.84 & 0 & 10 & $1.67(1.38-2.02)$ & 0.06 & 16.38 & 45.0 \\
\hline \multicolumn{11}{|l|}{ Geographic region } \\
\hline Asia & 6 & $1.19(0.98-1.45)$ & 0.06 & 15.00 & 46.7 & 7 & $1.48(1.26-1.74)$ & 0.43 & 8.07 & 0.9 \\
\hline Non-Asia & 9 & $1.14(1.05-1.25)$ & 0.43 & 12.20 & 1.7 & 13 & $1.58(1.40-1.80)$ & 0.22 & 22.38 & 19.6 \\
\hline \multicolumn{11}{|l|}{ BMI ascertainment } \\
\hline Self-reported & 7 & $1.18(1.01-1.36)$ & 0.46 & 9.74 & 0 & 7 & $1.65(1.32-2.05)$ & 0.20 & 12.16 & 26.0 \\
\hline \multicolumn{11}{|c|}{ Adjustment for confounders smoking } \\
\hline Yes & 10 & $1.16(1.02-1.31)$ & 0.16 & 20.42 & 26.5 & 11 & $1.55(1.31-1.83)$ & 0.21 & 19.06 & 21.3 \\
\hline No & 5 & $1.14(0.98-1.32)$ & 0.21 & 7.10 & 29.5 & 9 & $1.59(1.40-1.80)$ & 0.32 & 12.61 & 12.8 \\
\hline \multicolumn{11}{|l|}{ Alcohol intake } \\
\hline Yes & 7 & $1.27(1.10-1.47)$ & 0.37 & 10.87 & 8.0 & 7 & $1.64(1.31-2.07)$ & 0.15 & 13.31 & 32.4 \\
\hline No & 8 & $1.08(0.98-1.19)$ & 0.25 & 12.59 & 20.6 & 13 & $1.56(1.40-1.73)$ & 0.36 & 18.44 & 7.8 \\
\hline
\end{tabular}

$\mathrm{RR}$, relative risk; $\mathrm{CI}$, confidence interval; $\mathrm{BMI}$, body mass index; $p_{\mathrm{h}}, p$-value for heterogeneity; $Q$, Cochran's $\mathrm{Q}$ statistics. 
In stratified analysis by study design, a statistically significant positive association between BMI and GBC risk was observed for the cohort studies (overweight: SRRs $=1.15,95 \% \mathrm{CI}=1.02-1.29$; obesity: $\mathrm{SRRs}=1.62,95 \% \mathrm{CI}=1.45-1.81$ ). Moreover, in cohort studies with follow-up time $>10$ years, overweight and obesity were strongly associated with incidence of GBC (overweight: SRRs $=1.12$, $95 \%$ CI $=1.00-1.27$; obesity: SRRs $=1.65,95 \% \mathrm{CI}=1.49-1.83)$, while only overweight was observed associated with GBC risk in cohort studies with follow-up time $<10$ years (SRRs $=1.52$, $95 \% \mathrm{CI}=1.06-2.19$ ). For the case-control studies, only obesity was strongly associated with GBC risk (SRRs $=1.37,95 \% \mathrm{CI}=1.10-1.71)$. The SRRs of GBC incidence for obesity in population-based case-control studies was $1.43(95 \% \mathrm{CI}=1.09-1.89)$; no significant association between obesity and GBC risk was observed in hospital-based case-control studies.

A significant gender-specific difference was observed in the association between obesity and GBC risk, and obese women had a higher risk of GBC (women: $\mathrm{SRRs}=1.67,95 \% \mathrm{CI}=1.38-2.02$; men: $\mathrm{SRRs}=1.42,95 \% \mathrm{CI}=1.21-1.66)$. However, overweight men are not associated with risk of GBC (SRRs $=1.06,95 \% \mathrm{CI}=0.94-1.20)$.

In stratified analysis by geographic location, the association between obesity and the risk of GBC was similar for both Asia and non-Asia (Table 3). For non-Asians, overweight was strongly associated with GBC incidence (SRRs $=1.14,95 \% \mathrm{CI}=1.05-1.25$; no significant association between overweight and the GBC risk was observed for Asians. In stratified analysis by BMI ascertainment, both overweight and obesity had a higher risk of GBC in self-report studies

In addition, when stratified by potential confounders, overweight people with smoking and alcohol consumption were strongly associated GBC risk, no significant association between overweight and the risk of GBC was found in those without smoking and alcohol consumption (non-smokers: SRRs $=1.14,95 \% \mathrm{CI}=0.98-1.32$; non-alcoholics: SRRs $=1.08,95 \% \mathrm{CI}=0.98-1.19$ ), indicating that smoking and alcohol consumption are positive confounders. No differences were observed in the association between obesity and GBC incidence when stratified by smoking and alcohol consumption.

\subsection{Sensitivity Analyses and Publication Bias}

In the sensitivity analyses, we removed one study at a time and calculated the SRRs. We found that there were no changes in the direction of effect when any one study was excluded, supporting the robustness of our results. For example, when the study of Engeland et al. [19] was excluded (which seemed to have a strong influence on the estimate of effect), the SRR remained similar with the overall pooled RRs (SRRs $=1.15,95 \% \mathrm{CI}=1.03-1.28, I^{2}=17.8 \%$ ).

No indication of publication bias was observed in the literature on BMI and GBC risk in overweight group based on the Egger's test $(p=0.483)$ results (Figure $3 \mathrm{~A})$. For BMI and GBC risk in the obesity group, the funnel plot showed a little asymmetry (Figure 3B), indicating some evidence of bias. However, when the "trim and fill" approach was performed, data was unchanged, suggesting that the effect of publication bias could be negligible. 


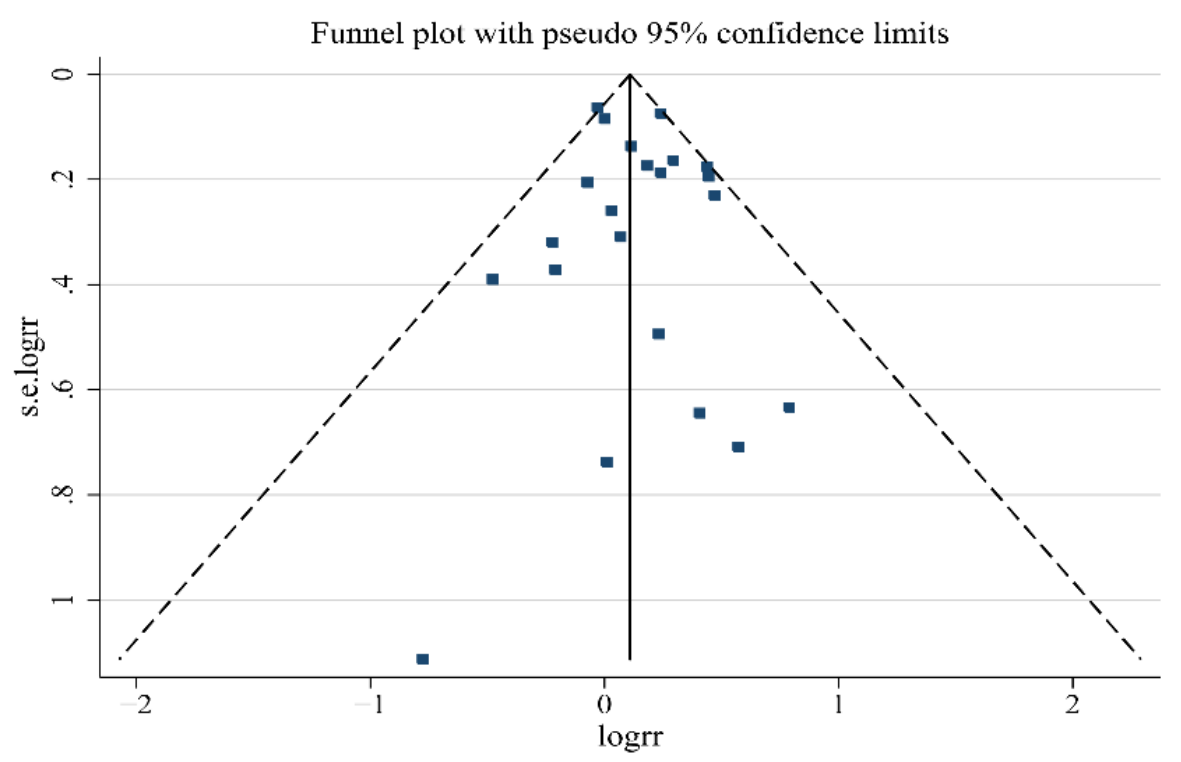

(A)

Funnel plot with pseudo $95 \%$ confidence limits

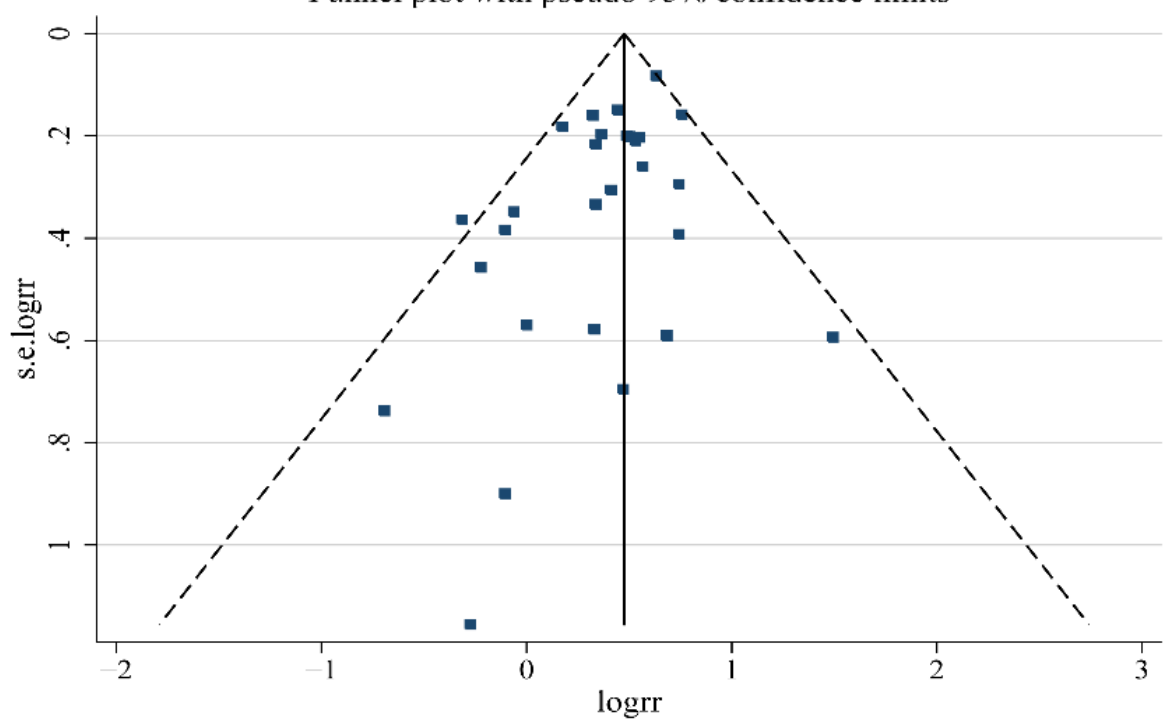

(B)

Figure 3. (A) Funnel plot of studies evaluating the association between overweight and gallbladder cancer risk ( $p=0.483)$; (B) Funnel plot of studies evaluating the association between obesity and gallbladder cancer risk $(p=0.019)$.

\section{Discussion and Conclusions}

In this meta-analysis, we found that overweight and obesity were associated with $14 \%$ and $56 \%$ excess risk of GBC, respectively. Our results are consistent with previous studies that the association between obesity and GBC risk was higher in women than men. Women with overweight had a higher risk of GBC, while no significant association between overweight and the GBC risk was observed for men.

Several biological mechanisms were hypothesized for the possible carcinogenesis of gallbladder associated with excess body weight. Obesity is often accompanied with metabolic syndrome, characterized by insulin resistance, hyperglycemia, dyslipidemias, and hypertension [33]. In obese adults, alterations occur in the circulating levels of insulin, insulin-like growth factor (IGF)-1, adipokines, inflammatory factors, and pro-inflammatory cytokines. These mediators associated 
with the obesity, contribute to cancer-related processes, including growth signaling, inflammation, and vascular alterations [34]. Furthermore, obesity and metabolic syndrome are risk factors for gallstone disease [35], which may indirectly increase the risk of GBC [36]. In addition, female sex hormones adversely influence hepatic bile secretion and gallbladder function [37]. Estrogens increase cholesterol secretion and diminish bile salt secretion, while progestins act by reducing bile salt secretion and impairing gallbladder emptying leading to stasis [38]. These may partially explain the stronger association observed with overweight or obesity in women than in men.

Our meta-analysis has several strengths. (1) This meta-analysis was based on 20 epidemiologic studies, which might minimize the possibility of selection bias. (2) Most of the included studies provided more than one RRs, which could be applicable to accurately subgroup analysis. (3) The included studies evaluated multiple confounders including smoking and alcohol. The relationships between BMI and risk of GBC in each study were derived from regression after adjustment at least for age and gender.

Our meta-analysis has limitations that affect interpretation of the true results. First, inadequate control for confounders may bias the results, leading to exaggeration or underestimation of risk estimates. Thus, when interpreting the association between excess body weight and GBC risk, possible unmeasured or residual confounding factors should be considered. Five studies were of acceptable quality (NOS $<7$ ), mainly due to the adjustments made for confounders. Smoking and alcohol abuse is closely related to GBC risk. Subgroup analysis results also shown that overweight people with smoking and alcohol consumption were strongly associated GBC risk, while no significant association between overweight and the risk of GBC was found in those without smoking and alcohol consumption, suggesting that data from unadjusted studies might lead to an overestimation of overweight in the development of GBC. Interestingly, no differences were observed in the association between obesity and GBC incidence when stratified by smoking and alcohol consumption, suggesting that obesity might be an independent risk factor. Gallstone is closely related to GBC risk [39]. Meanwhile, obesity tends to be accompanied with DM, which is also associated with increased GBC risk [4,40]. However, most studies did not adjust for these risk factors. This could have led to an overestimation of the true association between obesity and risk of GBC. Second, although BMI is the most commonly used anthropometric tool to assess relative weight and classify obesity, BMI cannot make the distinction between an excess body weight due to high levels of fat mass or muscle mass. Generally, an excess fat mass is more frequently associated with metabolic syndrome than a high level of muscle mass, leading to increased risk of GBC. Furthermore, obese individuals differ in regional body fat distribution. Adipose tissue now is considered as an endocrine organ, playing an important role in tumor microenvironment. Abdominal adiposity might play a more important role than peripheral type of obesity in the development of abdominal cancer. Other tools, such as waist circumference (WC), waist-to-height ratio (WHtR), and waist-to-hip ratio (WHR), which are more useful than BMI in determining abdominal adiposity, might be more sensitive in predicting the risk of abdominal cancer. However, little clinical evidence can be achieved to compare the screening potential of each tool. Third, several studies in this meta-analysis relied on self-reported weight and height data, which may attenuate the relative risk estimates. However, the SRRs for BMI ascertained by measurement were similar to those by self-reported. Finally, as in any meta-analysis, the possibility of publication bias is of concern, because a few studies with null results tend not to be published. However, the results from this study did not provide evidence for such a bias.

There was significant heterogeneity observed across studies about overweight and GBC risk, but the heterogeneity is low and acceptable with $I^{2}=31.9 \%$, so we could combine studies in a meta-analysis. We analyzed this review in both fixed effects and random effects, and found that they had no significant differences. Thus, the more conservative one, random effects, was chosen finally. Next, when we tried to carry out subgroup analysis to investigate sources of heterogeneity, statistical heterogeneity was lower in analysis of case-control studies, population based studies, 
Non-Asia studies and studies of BMI ascertainment by self-report, indicating that these might account for heterogeneity observed in studies about overweight and GBC risk.

In summary, findings of this meta-analysis provide evidence that excess body weight may increase GBC risk. Further studies that meet strict criteria on this subject are needed to strengthen the association between BMI and GBC risk, especially those adjusting potential confounding factors such as gallstones and DM.

Acknowledgments: This work was supported by grants from the National Natural Science Foundation of China (No. 81271876), Natural Science Foundation of Shandong Province (No. ZR2011HL004), Jining Science and Technology Project (No. 2014JNNK21-Liu Ning, 2010-Tan Wenbin), Program for Innovation of Graduate Education of Shandong Province (No. SDYY14014).

Author Contributions: Wenbin Tan and Min Gao conceived and designed the study. Wenbin Tan and Min Gao performed a literature search and identified eligible studies. Guoan Zhang, Tong Xu and Wen Cui extracted data from retrieved studies. Ning Liu carried out statistical analysis and interpreted results. The authors do not have any possible conflicts of interest. All drafts of the reports were written by Wenbin Tan and Min Gao. All authors read and approved the final paper.

Conflicts of Interest: The authors declare no conflict of interest.

\section{Abbreviations}

$\begin{array}{ll}\text { GBC } & \text { Gallbladder cancer } \\ \text { BMI } & \text { Body mass index } \\ \text { OR } & \text { Odds ratio } \\ \text { RR } & \text { Relative risk } \\ \text { HR } & \text { Hazard ratio } \\ \text { SRRs } & \text { Summary relative risks } \\ \text { CI } & \text { Confidence intervals } \\ \text { DM } & \text { Diabetes mellitus }\end{array}$

\section{References}

1. Misra, S.; Chaturvedi, A.; Misra, N.C.; Sharma, I.D. Carcinoma of the gallbladder. Lancet Oncol. 2003, 4, 167-176. [CrossRef]

2. Foster, J.M.; Hoshi, H.; Gibbs, J.F.; Iyer, R.; Javle, M.; Chu, Q.; Kuvshinoff, B. Gallbladder cancer: Defining the indications for primary radical resection and radical re-resection. Ann. Surg. Oncol. 2007, 14, 833-840. [CrossRef] [PubMed]

3. Hsing, A.W.; Bai, Y.; Andreotti, G.; Rashid, A.; Deng, J.; Chen, J.; Goldstein, A.M.; Han, T.Q.; Shen, M.C.; Fraumeni, J.F., Jr.; et al. Family history of gallstones and the risk of biliary tract cancer and gallstones: A population-based study in Shanghai, China. Int. J. Cancer 2007, 121, 832-838. [CrossRef] [PubMed]

4. Jamal, M.M.; Yoon, E.J.; Vega, K.J.; Hashemzadeh, M.; Chang, K.J. Diabetes mellitus as a risk factor for gastrointestinal cancer among American veterans. World J. Gastroenterol. 2009, 15, 5274-5278. [CrossRef] [PubMed]

5. Hou, L.; Xu, J.; Gao, Y.T.; Rashid, A.; Zheng, S.L.; Sakoda, L.C.; Shen, M.C.; Wang, B.S.; Deng, J.; Han, T.Q.; et al. CYP17 MspA1 polymorphism and risk of biliary tract cancers and gallstones: A population-based study in Shanghai, China. Int. J. Cancer 2006, 118, 2847-2853. [CrossRef] [PubMed]

6. Ji, J.; Hemminki, K. Variation in the risk for liver and gallbladder cancers in socioeconomic and occupational groups in Sweden with etiological implications. Int. Arch. Occup. Environ. Health 2005, 78, 641-649. [CrossRef] [PubMed]

7. Moerman, C.J.; Bueno, D.M.H.; Runia, S. Smoking, alcohol consumption and the risk of cancer of the biliary tract; a population-based case-control study in The Netherlands. Eur. J. Cancer Prev. 1994, 3, 427-436. [CrossRef] [PubMed]

8. Wolk, A.; Gridley, G.; Svensson, M.; Nyren, O.; McLaughlin, J.K.; Fraumeni, J.F.; Adam, H.O. A prospective study of obesity and cancer risk (Sweden). Cancer Causes Control 2001, 12, 13-21. [CrossRef] [PubMed]

9. Moller, H.; Mellemgaard, A.; Lindvig, K.; Olsen, J.H. Obesity and cancer risk: A Danish record-linkage study. Eur. J. Cancer 1994, 30, 344-350. [CrossRef] 
10. Strom, B.L.; Soloway, R.D.; Rios-Dalenz, J.L.; Rodriguez-Martinez, H.A.; West, S.L.; Kinman, J.L.; Polansky, M.; Berlin, J.A. Risk factors for gallbladder cancer. An international collaborative case-control study. Cancer 1995, 76, 1747-1756. [CrossRef]

11. Hemminki, K.; Li, X.; Sundquist, J.; Sundquist, K. Obesity and familial obesity and risk of cancer. Eur. J. Cancer Prev. 2011, 20, 438-443. [CrossRef] [PubMed]

12. Alvi, A.R.; Siddiqui, N.A.; Zafar, H. Risk factors of gallbladder cancer in Karachi-a case-control study. World J. Surg. Oncol. 2011, 9, 164. [CrossRef] [PubMed]

13. Stroup, D.F.; Berlin, J.A.; Morton, S.C.; Olkin, I.; Williamson, G.D.; Rennie, D.; Moher, D.; Becker, B.J.; Sipe, T.A.; Thacker, S.B. Meta-analysis of observational studies in epidemiology: A proposal for reporting. JAMA 2000, 283, 2008-2012. [CrossRef] [PubMed]

14. Wells, G.A.; Shea, B.; O'Connell, D.; Peterson, J.; Welch, V.; Losos, M.; Tugwell, P. The Newcastle-Ottawa Scale (NOS) for Assessing the Quality of Nonrandomised Studies in Meta-Analyses; Ottawa Hospital Research Institute: Ottawa, ON, Canada, 2013.

15. Higgins, J.P.; Thompson, S.G. Quantifying heterogeneity in a meta-analysis. Stat. Med. 2002, 21, $1539-1558$. [CrossRef] [PubMed]

16. Sterne, J.A.; Egger, M. Funnel plots for detecting bias in meta-analysis: Guidelines on choice of axis. J. Clin. Epidemiol. 2001, 54, 1046-1055. [CrossRef]

17. Duval, S.; Tweedie, R. Trim and fill: A simple funnel-plot-based method of testing and adjusting for publication bias in meta-analysis. Biometrics 2000, 56, 455-463. [CrossRef] [PubMed]

18. Calle, E.E.; Rodriguez, C.; Walker-Thurmond, K.; Thun, M.J. Overweight, obesity, and mortality from cancer in a prospectively studied cohort of U.S. adults. N. Engl. J. Med. 2003, 348, 1625-1638. [CrossRef] [PubMed]

19. Engeland, A.; Tretli, S.; Austad, G.; Bjorge, T. Height and body mass index in relation to colorectal and gallbladder cancer in two million Norwegian men and women. Cancer Causes Control 2005, 16, 987-996. [CrossRef] [PubMed]

20. Ishiguro, S.; Inoue, M.; Kurahashi, N.; Iwasaki, M.; Sasazuki, S.; Tsugane, S. Risk factors of biliary tract cancer in a large-scale population-based cohort study in Japan (JPHC study); with special focus on cholelithiasis, body mass index, and their effect modification. Cancer Causes Control 2008, 19, 33-41. [CrossRef] [PubMed]

21. Jee, S.H.; Yun, J.E.; Park, E.J.; Cho, E.R.; Park, I.S.; Sull, J.W.; Ohrr, H.; Samet, J.M. Body mass index and cancer risk in Korean men and women. Int. J. Cancer 2008, 123, 1892-1896. [CrossRef] [PubMed]

22. Kuriyama, S.; Tsubono, Y.; Hozawa, A.; Shimazu, T.; Suzuki, Y.; Koizumi, Y.; Suzuki, Y.; Ohmori, K.; Nishino, Y.; Tsuji, I. Obesity and risk of cancer in Japan. Int. J. Cancer 2005, 113, 148-157. [CrossRef] [PubMed]

23. Oh, S.W.; Yoon, Y.S.; Shin, S.A. Effects of excess weight on cancer incidences depending on cancer sites and histologic findings among men: Korea National Health Insurance Corporation Study. J. Clin. Oncol. 2005, 23, 4742-4754. [CrossRef] [PubMed]

24. Samanic, C.; Chow, W.H.; Gridley, G.; Jarvholm, B.; Fraumeni, J.J. Relation of body mass index to cancer risk in 362,552 Swedish men. Cancer Causes Control 2006, 17, 901-909. [CrossRef] [PubMed]

25. Samanic, C.; Gridley, G.; Chow, W.H.; Lubin, J.; Hoover, R.N.; Fraumeni, J.J. Obesity and cancer risk among white and black United States veterans. Cancer Causes Control 2004, 15, 35-43. [CrossRef] [PubMed]

26. Song, Y.M.; Sung, J.; Ha, M. Obesity and risk of cancer in postmenopausal Korean women. J. Clin. Oncol. 2008, 26, 3395-3402. [CrossRef] [PubMed]

27. Grainge, M.J.; West, J.; Solaymani-Dodaran, M.; Aithal, G.P.; Card, T.R. The antecedents of biliary cancer: A primary care case-control study in the United Kingdom. Br. J. Cancer 2009, 100, 178-180. [CrossRef] [PubMed]

28. Hsing, A.W.; Sakoda, L.C.; Rashid, A.; Chen, J.; Shen, M.C.; Han, T.Q.; Wang, B.S.; Gao, Y.T. Body size and the risk of biliary tract cancer: A population-based study in China. Br. J. Cancer 2008, 99, 811-815. [CrossRef] [PubMed]

29. Machova, L.; Cizek, L.; Horakova, D.; Koutna, J.; Lorenc, J.; Janoutova, G.; Janout, V. Association between obesity and cancer incidence in the population of the District Sumperk, Czech Republic. Onkologie 2007, 30, 538-542. [CrossRef] [PubMed]

30. Nakadaira, H.; Lang, I.; Szentirmay, Z.; Hitre, E.; Kaster, M.; Yamamoto, M. A case-control study of gallbladder cancer in Hungary. Asian Pac. J. Cancer Prev. 2009, 10, 833-836. [PubMed] 
31. Serra, I.; Yamamoto, M.; Calvo, A.; Cavada, G.; Baez, S.; Endoh, K.; Watanabe, H.; Tajima, K. Association of chili pepper consumption, low socioeconomic status and longstanding gallstones with gallbladder cancer in a Chilean population. Int. J. Cancer 2002, 102, 407-411. [CrossRef] [PubMed]

32. Zatonski, W.A.; Lowenfels, A.B.; Boyle, P.; Maisonneuve, P.; Bueno, D.M.H.; Ghadirian, P.; Jain, M.; Przewozniak, K.; Baghurst, P.; Moerman, C.J.; et al. Epidemiologic aspects of gallbladder cancer: A case-control study of the SEARCH Program of the International Agency for Research on Cancer. J. Natl. Cancer Inst. 1997, 89, 1132-1138. [CrossRef] [PubMed]

33. Hursting, S.D.; Hursting, M.J. Growth signals, inflammation, and vascular perturbations: Mechanistic links between obesity, metabolic syndrome, and cancer. Arterioscler. Thromb. Vasc. Biol. 2012, 32, 1766-1770. [CrossRef] [PubMed]

34. Dali-Youcef, N.; Mecili, M.; Ricci, R.; Andres, E. Metabolic inflammation: Connecting obesity and insulin resistance. Ann. Med. 2013, 45, 242-253. [CrossRef] [PubMed]

35. Shebl, F.M.; Andreotti, G.; Meyer, T.E.; Gao, Y.T.; Rashid, A.; Yu, K.; Shen, M.C.; Wang, B.S.; Han, T.Q.; Zhang, B.H.; et al. Metabolic syndrome and insulin resistance in relation to biliary tract cancer and stone risks: A population-based study in Shanghai, China. Br. J. Cancer 2011, 105, 1424-1429. [CrossRef] [PubMed]

36. Randi, G.; Franceschi, S.; La Vecchia, C. Gallbladder cancer worldwide: Geographical distribution and risk factors. Int. J. Cancer 2006, 118, 1591-1602. [CrossRef] [PubMed]

37. Cirillo, D.J.; Wallace, R.B.; Rodabough, R.J.; Greenland, P.; LaCroix, A.Z.; Limacher, M.C.; Larson, J.C. Effect of estrogen therapy on gallbladder disease. JAMA 2005, 293, 330-339. [CrossRef] [PubMed]

38. Gabbi, C.; Kim, H.J.; Barros, R.; Korach-Andre, M.; Warner, M.; Gustafsson, J.A. Estrogen-dependent gallbladder carcinogenesis in LXRbeta-/- female mice. Proc. Natl. Acad. Sci. USA 2010, 107, 14763-14768. [CrossRef] [PubMed]

39. Stinton, L.M.; Shaffer, E.A. Epidemiology of gallbladder disease: Cholelithiasis and cancer. Gut Liver 2012, 6, 172-187. [CrossRef] [PubMed]

40. Ren, H.B.; Yu, T.; Liu, C.; Li, Y.Q. Diabetes mellitus and increased risk of biliary tract cancer: Systematic review and meta-analysis. Cancer Causes Control 2011, 22, 837-847. [CrossRef] [PubMed]

(C) 2015 by the authors; licensee MDPI, Basel, Switzerland. This article is an open access article distributed under the terms and conditions of the Creative Commons by Attribution (CC-BY) license (http:/ / creativecommons.org/licenses/by/4.0/). 